\title{
Idosos octogenários nos contextos urbano e rural: comparação socioeconômica, morbidades e qualidade de vida
}

\author{
Octogenarians in rural and urban settings: socioeconomic comparison, morbidities \\ and quality of life
}

\author{
Octogenarios en los contextos rural y urbano: comparación socioeconómica, \\ morbilidades y calidad de vida
}

\author{
Darlene Mara dos Santos Tavares'; Amanda Gonçalves Ribeiro II; Pollyana Cristina dos Santos Ferreira ${ }^{\text {III; }}$ \\ Nayara Paula Fernandes Martins ${ }^{I V}$; Maycon Sousa Pegorari ${ }^{\mathrm{V}}$
}

\begin{abstract}
RESUMO: Estudo transversal, observacional e analítico que objetivou comparar as variáveis socioeconômicas, morbidades autorreferidas e qualidade de vida de 326 octogenários da zona urbana e 74 da rural, residentes em Uberaba. A coleta de dados ocorreu em 2008 e 2010, utilizando-se os instrumentos: BOMFAQ, WHOQOL-BREF e WHOQOL-OLD. Aplicou-se análise descritiva, testes qui-quadrado e t-Student e regressões logística e linear múltipla $(p<0,05)$. Predominaram mulheres octogenárias na zona urbana e homens na zona rural. O estado conjugal e a escolaridade permaneceram associados ao local de moradia. Os octogenários da zona urbana apresentaram piores condições de saúde e escores inferiores nos domínio físico e relações sociais e nas facetas autonomia e atividades passadas, presentes e futuras; enquanto os da zona rural obtiveram escores inferiores no domínio meio ambiente e na faceta funcionamento dos sentidos. Esses resultados podem subsidiar ações de saúde específicas à essa clientela, considerando as peculiaridades do ambiente em que vivem.

Palavras-Chave: Idoso de 80 anos ou mais; população urbana; população rural; saúde do idoso.
\end{abstract}

\begin{abstract}
This cross-sectional, observational, analytical study compared socioeconomic variables, self-reported morbidity and quality of life of octogenarians resident in Uberaba, 326 in the urban and 74 in the rural area. Data was collected in 2008 and 2010, using BOMFAQ, WHOQOL-BREF and WHOQOL-OLD. Descriptive analysis, chi-square and Student t-tests, and multiple linear and logistic regressions $(p<0.05)$ were applied. Octogenarians were predominantly women in urban areas and men in rural areas. Marital status and education were associated with place of residence. Octogenarians in the urban area had poorer health and scored lower in the physical and social relations domains and in the autonomy and past, present and future activities facets, while rural octogenarians scored lower in the environment domain and sensorial functioning facet. These results may support specific health measures for this population, considering the peculiarities of the environment they live in. Keywords: Elderly, 80 and over; urban population; rural population; health of the elderly.
\end{abstract}

RESUMEN: Estudio transversal, observacional y analítico que tiene como objetivo comparar las variables socioeconómicas, morbilidades autorreferidas y calidad de vida de 326 octogenarios de la región urbana y 74 de la rural, residentes en Uberaba. Los datos fueron recolectados en 2008 y 2010, utilizando los instrumentos: BOMFAQ, WHOQOL-BREF y WHOQOL-OLD. Se ha aplicado análisis descriptivo, testes chi-cuadrado, t-Student, regresión lineal múltiple y logística $(p<0,05)$. Mujeres octogenarias predominaron en zonas urbanas y los hombres en zona rural. El estado civil y la educación estaban asociados con el lugar de residencia. Los octogenarios del área urbana tienen peores condiciones de salud y las puntuaciones más bajas en las relaciones físicas y sociales, la autonomía y las actividades pasadas, presentes y futuras, mientras que los de la zona rural obtienen puntuaciones más bajas en el dominio del medio ambiente y en cuanto al funcionamiento de los sentidos. Estos resultados pueden subsidiar acciones de salud específicas para esta población, teniendo en cuenta las peculiaridades del entorno donde viven. Palabras Clave: Anciano de 80 años o más; población urbana; población rural; salud del anciano.

\section{INTRODUÇÃo}

O envelhecimento populacional tem ocorrido a um nível acelerado. Estima-se que nas próximas duas décadas, o número de idosos ultrapasse 30 milhões de pessoas, representando $13 \%$ da população mundial ${ }^{1}$. No Brasil, considerando o período entre 1999 a 2009, verificou-se um aumento percentual dessa faixa etária,

'Enfermeira. Doutora em Enfermagem. Professora Associada do Departamento de Enfermagem em Educação e Saúde Comunitária do Curso de Graduação em Enfermagem. Universidade Federal do Triângulo Mineiro. Uberaba, Minas Gerais, Brasil. E-mail: darlenetavares@enfermagem.uftm.edu.br.

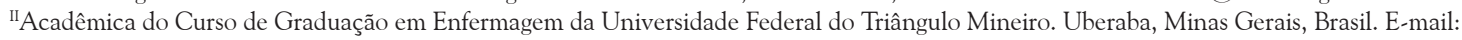
mandy.ribeiro93@hotmail.com.

IIIEnfermeira. Mestre em Atenção à Saúde. Professora Substituta da Universidade Federal do Triângulo Mineiro. Professora do Departamento de Medicina Social. Pesquisadora do Grupo de Pesquisa em Saúde Coletiva. Uberaba, Minas Gerais, Brasil. E-mail: pollycris21@bol.com.br.

${ }^{I V}$ Enfermeira. Mestre em Atenção à Saúde pela Universidade Federal do Triângulo Mineiro. Professora substituta da Universidade Federal do Triângulo Mineiro. Professora do Departamento de Medicina Social. Uberaba, Minas Gerais, Brasil. E-mail: nayara.pfmartins@gmail.com.

vFisioterapeuta. Mestre em Atenção à Saúde pela Universidade Federal do Triângulo Mineiro. Pesquisador do Grupo de Pesquisa em Saúde Coletiva. Uberaba, Minas Gerais, Brasil. E-mail: mayconpegorari@yahoo.com.br. 
passando de 9,1\% para 11,3\% em relação à população total, correspondendo, em 2009, a 21 milhões de pessoas com 60 anos ou mais ${ }^{2}$.

$\mathrm{O}$ aumento da expectativa de vida também se constitui como fenômeno mundial, ressaltando-se que indivíduos com 80 anos ou mais representam a faixa etária que mais cresce no mundo ${ }^{3}$, podendo chegar a $15 \%$ da população em 20202. No Brasil, entre os idosos, 14,4\% são octogenários, representando 1,5\% da população. Em Uberaba-MG, local de interesse dessa pesquisa o percentual de octogenários corresponde a $1,6 \%$, acima da média nacional ${ }^{4}$.

Tal cenário tem implicado em discussões, entre pesquisadores da saúde e promotores de políticas sociais, sobre os desafios que o aumento da longevidade tem imposto à maioria das sociedades, tanto as desenvolvidas quanto as em desenvolvimento ${ }^{5}$.

Nessa perspectiva, um fator que tem sido investigado é o ambiente onde o idoso reside. Porém percebe-se, ainda, a necessidade da realização de pesquisas que ampliem o conhecimento sobre a temática do envelhecimento em ambientes rurais, em comparação à zona urbana, para poder atender de forma mais igualitária aos anseios de um maior número de idosos brasileiros ${ }^{6}$.

Desse modo, esse estudo teve como objetivo comparar as variáveis socioeconômicas, as morbidades autorreferidas e a qualidade de vida (QV) de octogenários residentes nas zonas urbana e rural de um município de Minas Gerais.

\section{REVISÃO DE LITERATURA}

O envelhecimento da população traz consigo a necessidade de um olhar voltado para a saúde dessa faixa etária. Os indicadores sociais e demográficos vêm demonstrando que o grupo de idosos cresce expressivamente, dessa forma novas exigências no contexto das políticas públicas de saúde tornam-se necessárias para o atendimento a essa clientela ${ }^{2}$.

A literatura científica evidencia que a população que vive na zona rural envelhece à semelhança dos que vivem na zona urbana; entretanto, aponta para uma realidade em que predomina a pobreza, o isolamento geográfico, baixos níveis educacionais, residências mais precárias, limitações de transporte, problemas crônicos de saúde e distância dos recursos sociais e de saúde ${ }^{5}$.

Estudo realizado com idosos octogenários da zona rural de Encruzilhada do Sul- RS observou um percentual de $10,6 \%$ de idosos com 80 anos ou mais de idade. Destes, a maioria era do sexo feminino, viúvos e aposentados. Entre as morbidades autorreferidas destacaram-se a hipertensão arterial sistêmica, problemas de coluna, reumatismo, insônia e catarata ${ }^{5}$.
Em relação à presença de morbidades, sabe-se que conforme aumenta a idade, maiores são as chances de o idoso adquirir alguma doença crônica ${ }^{2}$, podendo repercutir negativamente em sua QV. Dados do Instituto Brasileiro de Geografia e Estatística (IBGE) mostram que apenas $22,6 \%$ das pessoas com 60 anos ou mais referem não apresentar alguma doença, reduzindo para $19,7 \%$, entre aqueles com 75 anos ou mais de idade 2 .

A QV também tem sido alvo de estudos no Brasil. Ao compará-la entre idosos que residiam nas zonas urbana e rural, pesquisa realizada na Paraíba verificou que as condições de vida nesses dois ambientes, embora diferentes, não influenciam na QV desses idosos. Contudo, a falta de recursos financeiros foi associada aos menores escores de QV entre aqueles com menor poder aquisitivo ${ }^{6}$.

Apesar de iniciativas de estudos com idosos em idade avançada, verifica-se ainda escassez de produção de conhecimento a respeito das condições de saúde de idosos longevos ${ }^{7}$.

Ressalva-se que compreender as condições de saúde dos idosos mais velhos favorece intervenções direcionadas, de forma a atender a suas demandas e propiciar melhorias que possam impactar na QV?.

Assim, é relevante o estudo das condições de saúde de octogenários na zona urbana e rural, uma vez que o processo de vida e envelhecimento pode lhes conferir características peculiares, a partir dos diferentes contextos e realidades.

\section{Metodologia}

Esta pesquisa faz parte de dois projetos maiores conduzidos pelo Grupo de Pesquisa em Saúde Coletiva da Universidade Federal do Triângulo Mineiro (UFTM), com idosos das zonas urbana e rural do município de Uberaba-MG, nos anos de 2008 e 2011, respectivamente. Trata-se de estudos do tipo inquérito domiciliar, analítico, transversal e observacional.

$\mathrm{Na}$ zona urbana a amostra foi calculada com base em 2.892 idosos, considerando $95 \%$ de confiança, $80 \%$ de poder do teste, margem de erro de $4 \%$ para as estimativas intervalares e uma proporção estimada de $\pi=0,5$ para as proporções de interesse. Participaram, em 2008, 2.142 idosos.

Para compor a população da zona rural, foi obtido, em junho de 2010, junto às equipes de saúde da família (ESF), o número de idosos cadastrados de acordo com cada área de abrangência. A zona rural do referido município possui $100 \%$ de cobertura pela ESF, totalizando 1.297 idosos cadastrados. Ao término da coleta de dados, foram entrevistados 850 idosos.

Foram incluídos neste estudo idosos residentes nas zonas rural e urbana de Uberaba-MG, com 80 anos 
ou mais de idade e que não apresentaram declínio cognitivo, participando 326 idosos na zona urbana e 74 na rural.

O Miniexame do Estado Mental (MEEM) foi aplicado antes de iniciar a coleta dos dados a fim de realizar a avaliação cognitiva do idoso. Na zona urbana, foi utilizada a versão reduzida validada por pesquisadores do Projeto Saúde, Bem Estar e Envelhecimento $(\mathrm{SABE})^{8}$, e, na zona rural, utilizou-se o instrumento traduzido e validado no Brasil ${ }^{9}$. Ambos os instrumentos permitem rastrear a presença de declínio cognitivo, sendo considerada para estabelecer o ponto de corte, a escolaridade do idoso. A mudança de instrumento entre as duas localidades justifica-se devido ao fato de a coleta ter ocorrido em momentos distintos, sendo percebido pelos pesquisadores que o instrumento traduzido e validado no Brasil $^{9}$ seria mais adequado para realizar a avaliação cognitiva da população residente na zona rural.

Utilizou-se parte do Questionário Brasileiro de Avaliação Funcional e Multidimensional (BOMFAQ), adaptado à realidade brasileira ${ }^{10}$, para obtenção das variáveis socioeconômicas e morbidades autorreferidas.

Para a avaliação da QV, utilizaram-se as versões validadas no Brasil do World Health Organization Quality of Life - BREF (WHOQOL-BREF) ${ }^{11}$ e do World Health Organization Quality of Life Assessment for Older Adults (WHOQOL-OLD) ${ }^{12}$.

As variáveis analisadas foram: sexo, idade, estado conjugal, escolaridade, arranjo domiciliar, renda individual mensal, morbidades autorreferidas, domínios da QV avaliada pelo WHOQOL-BREF (físico, psicológico, relações sociais e meio ambiente) e facetas do WHOQOL-OLD (funcionamento dos sentidos; autonomia; atividades passadas, presentes e futuras; participação social, morte e morrer e intimidade).

As entrevistas realizadas foram submetidas à revisão e à codificação. Foram construídos dois bancos de dados eletrônicos, no programa Excelß, um para a zona urbana e outro para a zona rural.

Os dados coletados foram processados em microcomputador por duas pessoas, em dupla entrada, para verificação da consistência dos dados. No caso de inconsistências entre as bases procedeu-se à correção por meio da consulta à entrevista original. Para o presente estudo, foram selecionados nos bancos de dados das zonas urbanas e rurais os idosos que atenderam aos critérios de inclusão, compondo-se, assim, um único banco de dados.

Os dados foram transportados para o programa Statistical Package for the Social Sciences (SPSS), versão 17.0, para proceder à análise. Realizou-se análise estatística por meio de distribuição de frequências simples, média e desvio padrão. Para a comparação das variáveis categóricas aplicou-se o teste qui-quadrado e para as variáveis numéricas, $t-$ Student $(\mathrm{p}<0,05)$. Cada domínio do WHOQOL-BREF e facetas WHOQOLOLD foram analisados isoladamente.
A fim de minimizar possíveis fatores de confundimento as variáveis que apresentaram associação significativa $(\mathrm{p}<0,05)$ com o local de moradia, foram ajustadas de acordo com o sexo (feminino e masculino) a partir do modelo de regressão logística múltipla para as variáveis socioeconômicas e morbidades, e regressão linear múltipla para as variáveis relacionadas à QV. Para essa análise todas as variáveis foram transformadas em dicotômicas e as associações consideradas significativas quando $\mathrm{p}<0,05$.

Os projetos foram submetidos ao Comitê de Ética em Pesquisa com Seres Humanos da UFTM e aprovados com os protocolos no 897 e no 1477, respectivamente. Foram apresentados os objetivos das pesquisas e esclarecidas as dúvidas. Após a assinatura do Termo de Consentimento Livre e Esclarecido pelo idoso, iniciou-se a entrevista.

\section{Resultados e Discussão}

Verificou-se maior percentual de mulheres na zona urbana $(62,9 \%)$, enquanto na zona rural predominaram os homens $(58,1 \%)$; sendo que a média de idade para os octogenários da zona urbana foi de 84,4 anos $(\mathrm{DP}=3,83)$ e na zona rural, 83,8 anos $(\mathrm{DP}=3,02)$.

Estudo conduzido com idosos octogenários e nonagenários, das zonas urbana e rural de SiderópolisSC, encontraram dados condizentes, em que a média de idade foi de 85,06 anos $^{13}$. Destaca-se que na zona rural a média de idade foi discretamente inferior aos da zona urbana. Na zona rural pode ocorrer limitação ao acesso dos serviços de saúde, devido a dificuldades com meios de transporte e isolamento geográfico. Essas dificuldades podem ser vivenciadas mais intensamente entre os octogenários nessas localidades, frente a maior fragilidade oriunda do processo de envelhecimento e aproximação da finitude da vida. Nesse caso, a família atua como principal fonte de apoio aos idosos ${ }^{5}$. A equipe de saúde que atua na zona rural deve estar atenta às especificidades dessa faixa etária e também estimular o vínculo entre os idosos e familiares ${ }^{14}$.

O predomínio de homens residentes na zona rural contradiz estudos nacionais realizados com octogenários que vivem nessas áreas, em que houve maior percentual de mulheres ${ }^{5,15}$. Observou-se durante a coleta de dados, embora não tenha sido foco desse estudo, que as mulheres migram, com maior frequência, com os filhos para a zona urbana quando estes buscam oportunidades de estudo e emprego. Ao contrário, os homens idosos permanecem trabalhando no campo a fim de prover a família, mesmo em idades mais avançadas. Esse fato poderia justificar, em parte, o maior percentual de homens na zona rural e de mulheres na zona urbana. Adicionalmente, o predomínio de mulheres na zona urbana pode ser reflexo 
da maior expectativa de vida entre o sexo feminino e por apresentarem condição de saúde mais frágil que os homens, requerendo o deslocamento para a zona urbana para estarem mais próximas dos serviços de saúde e de atendimento ao idoso ${ }^{14}$.

A comparação das variáveis socioeconômicas dos octogenários, segundo o local de moradia, após a análise ajustada por sexo, é mostrada na Tabela 1 .

Para as variáveis socioeconômicas, após o ajuste segundo o sexo, o estado conjugal $(\mathrm{OR}=0,51$; IC95\%: $0,29-0,91 ; \mathrm{p}=0,023)$ e a escolaridade $(\mathrm{OR}=2,15$; IC95\%: 1,28-3,63; $p=0,004)$ permaneceram associados ao local de moradia, conforme a Tabela 1 .

Observou-se maior proporção de idosos residentes na zona urbana que não possuíam companheiro em relação aos idosos residentes na zona rural. Destaca-se que em ambos os grupos o maior percentual residia acompanhado, tanto na zona urbana $(82,5 \%)$, quanto na zona rural $(81,1 \%)$, Tabela 1 . Resultados consoantes foram observados em pesquisa realizada com octogenários residentes no Rio Grande do Sul, em que houve maior proporção de idosos sem companheiro na zona urbana comparado aos da zona rural $(p=0,046)^{15}$. Essa situação pode estar relacionada ao fato de haver maior percentual de mulheres na zona urbana e de homens na zona rural. Em estudo realizado com octogenários em Encruzilhada do Sul - RS verificaram predomínio de mulheres viúvas e de homens casados, acompanhando a tendência nacional ${ }^{5}$.

É importante que os profissionais de saúde reconheçam a situação conjugal dos octogenários, a fim de identificar as redes de apoio no âmbito familiar. Além disso, é relevante verificar se os idosos que não possuem companheiro residem sozinhos e/ou com outros idosos, visto que nesta faixa etária os octogenários podem requerer maior acompanhamento, devido à situação de saúde.
Em relação ao nível de escolaridade, a proporção de idosos na zona rural que não possuíam escolaridade foi superior em relação aos da zona urbana. Pesquisa realizada com octogenários de Porto Alegre-RS também verificou maior proporção de idosos sem escolaridade na zona rural comparados aos da zona urbana $(p=0,022)^{15}$. É possível que esta diferença esteja relacionada às dificuldades de acesso à escola, vivenciadas na zona rural ${ }^{15}$, além de fatores culturais em que não se valorizava a educação escolar no passado ${ }^{16}$.

A maioria dos octogenários referiu possuir renda individual mensal, correspondendo a 93,6\% na zona urbana e a 95,9\% na zona rural, não sendo verificada diferença significativa entre os grupos, Tabela 1. Em contrapartida, estudo desenvolvido no Rio Grande do Sul verificou maior proporção de idosos sem renda na zona rural comparado aos da urbana $(p=0,017)^{14}$. A renda é um fator relevante a ser identificado, visto que pode limitar o acesso aos serviços de saúde e sociais, gerando um impacto na QV dos idosos ${ }^{16}$.

As morbidades referidas pelos octogenários, segundo o local de moradia e a análise ajustada por sexo, estão relacionadas na Tabela 2. Serão apresentados os resultados com associação estatisticamente significativa após o ajuste para a variável de controle.

Na comparação entre os grupos, a proporção de idosos na zona urbana que relataram ter má circulação $(\mathrm{OR}=0,27$; IC95\%: 0,15-0,48; $<<0,001)$, problemas cardíacos $(\mathrm{OR}=0,54 ; \mathrm{IC} 95 \%: 0,31-0,95 ; \mathrm{p}=0,033)$, prisão de ventre $(\mathrm{OR}=0,32$; IC95\%: 0,18-0,59; $\mathrm{p}<0,001)$ e problema de visão $(\mathrm{OR}=0,40$; IC95\%: $0,23-0,68 ; p<0,001)$ foi significativamente superior aos da zona rural, de acordo com a Tabela 2.

Estudo desenvolvido nas cidades de São Paulo-SP e em Barcelona, na Espanha, verificou que as alterações circulatórias estiveram entre as morbidades mais referidas pelos octogenários ${ }^{17}$. Nessa pesquisa, a maior

TABELA 1: Proporção de idosos segundo local de moradia e análise ajustada por sexo para as variáveis socioeconômicas. Uberaba, Minas Gerais, 2008 e 2011.

\begin{tabular}{|c|c|c|c|c|c|c|c|c|}
\hline \multirow[t]{2}{*}{ Variáveis } & \multicolumn{2}{|c|}{ Urbana } & \multicolumn{2}{|c|}{ Rural } & \multirow[t]{2}{*}{$\mathbf{X}^{2}$} & \multirow[t]{2}{*}{$\mathrm{p}$} & \multirow[t]{2}{*}{ OR } & \multirow[t]{2}{*}{$\mathrm{p}^{(*)}$} \\
\hline & $f$ & $\%$ & $f$ & $\%$ & & & & \\
\hline \multicolumn{9}{|l|}{ Estado Conjugal } \\
\hline Sem & 225 & 69 & 35 & 47,3 & 12,508 & $<0,001$ & 0,51 & $0,023^{(* *)}$ \\
\hline Com & 101 & 31 & 39 & 52,7 & & & & \\
\hline \multicolumn{9}{|c|}{ Arranjo de Moradia } \\
\hline Vive sozinho & 57 & 17,5 & 14 & 18,9 & 0,085 & 0,771 & - & - \\
\hline Acompanhado & 269 & 82,5 & 60 & 81,1 & & & & \\
\hline \multicolumn{9}{|l|}{ Escolaridade } \\
\hline Sem & 104 & 31,9 & 37 & 50 & 8,655 & 0,003 & 2,15 & $0,004^{(* *)}$ \\
\hline Com & 222 & 68,1 & 37 & 50 & & & & \\
\hline \multicolumn{9}{|l|}{ Renda } \\
\hline Sem & 21 & 6,4 & 3 & 4,1 & 0,668 & 0,414 & - & - \\
\hline Com & 305 & 93,6 & 71 & 95,9 & & & & \\
\hline
\end{tabular}

OR (Odds Ratio) para um intervalo de confiança (IC) de $95 \%$ para a razão de chances de prevalência; ${ }^{(*)} \mathrm{p}(\mathrm{Ajustado}$ por sexo); ${ }^{* * *}(\mathrm{p}<0,05)$ 
TABELA 2: Proporção das morbidades referidas pelos idosos octogenários segundo local de moradia e análise ajustada por sexo. Uberaba, Minas Gerais, 2008 e 2011.

\begin{tabular}{lcccccccc}
\hline Morbidades & \multicolumn{2}{c}{ Urbana } & \multicolumn{2}{c}{ Rural } & $\mathbf{X}^{2}$ & $\mathbf{p}$ & OR & $\mathbf{p}^{(*)}$ \\
\hline Reumatismo & $\mathbf{f}$ & $\mathbf{\%}$ & $\mathbf{f}$ & $\%$ & & & & \\
Artrite/Artrose & 84 & 26,4 & 26 & 37,1 & 3,25 & 0,071 & - & - \\
Osteoporose & 123 & 39,3 & 18 & 26,1 & 4,237 & 0,04 & 0,64 & 0,055 \\
Asma ou Bronquite & 90 & 28,4 & 12 & 16,7 & 4,169 & 0,041 & 0,67 & 0,058 \\
Tuberculose & 47 & 14,5 & 11 & 14,9 & 0,006 & 0,937 & - & - \\
Embolia & 1 & 0,3 & - & - & 0,41 & 1 & - & - \\
Hipertensão arterial & 2 & 0,6 & 3 & 4,1 & 3,332 & 0,068 & - & - \\
Má circulação (varizes) & 210 & 64,4 & 52 & 70,3 & 0,914 & 0,339 & - & - \\
Problemas cardíacos & 186 & 57,2 & 18 & 24,3 & 26,121 & $<0,001$ & 0,27 & $<0,001^{(* *)}$ \\
Diabetes & 145 & 44,9 & 22 & 30,1 & 5,315 & 0,021 & 0,54 & $0,033^{(* *)}$ \\
Obesidade & 47 & 14,5 & 10 & 13,7 & 0,028 & 0,866 & - & - \\
Acidente vascular encefálico & 25 & 7,7 & 5 & 6,8 & 0,072 & 0,788 & - & - \\
Parkinson & 16 & 4,9 & 4 & 5,4 & 0,031 & 0,861 & - & - \\
Incontinência urinária & 9 & 2,8 & 4 & 5,5 & 1,223 & 0,269 & - & - \\
Incontinência fecal & 59 & 18,1 & 13 & 17,6 & 0,012 & 0,915 & - & - \\
Prisão de ventre & 12 & 3,7 & 3 & 4,1 & 0,023 & 0,88 & - & - \\
Problemas para dormir & 154 & 47,2 & 17 & 23 & 14,511 & $<0,001$ & 0,32 & $<0,001^{(* *)}$ \\
Catarata & 130 & 39,9 & 34 & 45,9 & 0,918 & 0,338 & - & - \\
Glaucoma & 164 & 50,6 & 33 & 45,2 & 0,698 & 0,403 & - & - \\
Problemas de coluna & 34 & 10,6 & 4 & 5,6 & 1,725 & 0,189 & - & - \\
Problema renal & 198 & 60,7 & 40 & 54,1 & 1,118 & 0,29 & - & - \\
Sequela acidente/trauma & 40 & 12,4 & 13 & 17,8 & 1,511 & 0,219 & - & - \\
Tumores malignos & 53 & 16,3 & 15 & 20,5 & 0,777 & 0,378 & - & - \\
Tumores benignos & 7 & 2,2 & 1 & 1,4 & 0,217 & 0,641 & - & - \\
Problema de visão & 19 & 5,8 & 2 & 2,7 & 1,376 & 0,241 & - & - \\
\hline OR (O, & 250 & 77,2 & 42 & 56,8 & 12,835 & $<0,001$ & 0,4 & $<0,001^{(* *)}$ \\
\hline
\end{tabular}

OR (Odds Ratio) para um intervalo de confiança (IC) de 95\% para a razão de chances de prevalência; ${ }^{(*)} \mathrm{p}($ Ajustado por sexo); ${ }^{(* *)}(p<0,05)$.

proporção de octogenários da zona urbana que referiram problemas de circulação pode estar relacionada ao predomínio de mulheres nessa localidade, uma vez que a literatura científica tem evidenciado maior prevalência no sexo feminino e em idades mais avançadas ${ }^{18,19}$.

A maior proporção de octogenários da zona urbana que referiram constipação intestinal também pode ter relação com o maior percentual de mulheres. Pesquisa de base populacional desenvolvida em Pelotas-RS verificou maior prevalência de constipação intestinal entre as mulheres ${ }^{20}$. Outro fator que pode interferir é a baixa ingestão de alimentos laxativos, como algumas frutas e verduras ${ }^{21}$. Assim, é possível que a maior disponibilidade desses alimentos na zona rural possa ter contribuído para a menor proporção de idosos com constipação intestinal residentes nessa área.

Pressupõe-se que a proporção superior de octogenários com problemas cardíacos na zona urbana deva-se a maior exposição aos fatores de risco nessa localidade. Em pesquisa realizada em Anchieta-ES, que avaliou os fatores de risco para problemas cardiovasculares, verificaram prevalência superior de pessoas residentes na zona urbana que eram inativas fisicamente, consumiam refrigerantes cinco ou mais dias da semana e obesos ou com sobrepeso, comparados aos da zona rural ${ }^{22}$. Os problemas cardiovasculares podem interferir negativamente na vida das pessoas. Assim, os profissionais de saúde devem estar aptos para oferecerem atendimento, abrangendo não apenas os aspectos biológicos, mas também as necessidades psicológicas, favorecendo a elaboração de mecanismos de enfrentamento da doença ${ }^{23}$.

A proporção de octogenários com problemas de visão na zona urbana foi superior aos da zona rural. Os problemas de visão acometem frequentemente os idosos, sendo que cerca de $90 \%$ necessitam utilizar lentes para auxiliá-los a enxergar de forma adequada ${ }^{24}$. É possível que na zona rural, onde ocorre um distanciamento dos serviços de saúde, seja mais restrita a avaliação da visão desses idosos, podendo ocorrer o subdiagnóstico.

A Tabela 3 apresenta os escores de QV para os domínios do WHOQOL-BREF e as facetas do WHOQOL-OLD, segundo local de moradia e análise ajustada por sexo.

Em relação à QV mensurada pelo WHOQOLBREF, após o ajuste segundo o sexo, verificou-se que os octogenários residentes na zona urbana apresentaram 
escores médios significativamente inferiores nos domínio físico $(\beta=0,15 ; p=0,002)$ e relações sociais $(\beta=0,11$; $\mathrm{p}=0,025)$ em relação aos idosos da zona rural, conforme a Tabela 3. Em contrapartida, os octogenários da zona rural obtiveram escores médios significativamente inferiores aos da zona urbana no domínio meio ambiente $(\beta=-0,10$; $\mathrm{p}=0,036)$, Tabela 3 .

Os escores significativamente inferiores no domínio físico, entre os octogenários da zona urbana, podem apresentar relação com o fato da maior predisposição à fragilidade nessa faixa etária tornando difícil a permanência na zona rural ${ }^{14}$. Assim, pressupõe-se que os idosos que apresentem piores condições físicas procurem residir nos centros urbanos, para estarem mais próximos aos serviços de saúde e do apoio familiar.

Os octogenários da zona urbana também apresentaram escores significativamente inferiores no domínio relações sociais quando comparados aos da zona rural. Na zona urbana, normalmente, ocorre um menor estabelecimento de laços afetivos entre vizinhos e outras pessoas, o que pode acarretar em maior isolamento social entre aqueles em idade mais avançada ${ }^{6}$.

Quanto aos menores escores no domínio meio ambiente entre os octogenários da zona rural observou-se durante a coleta de dados que existe uma preocupação dos idosos em relação à questão da segurança, reflexo da falta de policiamento nessa localidade, deixando-os mais susceptíveis à violência. Esse fato pode ter influenciado para os resultados obtidos. Ademais, na zona rural evidenciam-se menores oportunidades de atividades de lazer e recreação, dificuldades com meios de transporte e acesso ao entretenimento nas cidades ${ }^{25,26}$, o que poderia ter contribuído para o impacto na QV, avaliado por este domínio.

No WHOQOL-OLD, mesmo após o ajuste para a variável sexo, os octogenários da zona rural apresentaram escores médios significativamente inferiores aos da zona urbana na faceta funcionamento dos sentidos $(\beta=-0,11 ; p=0,029)$. Porém, aqueles que residem na zona urbana apresentaram escores médios inferiores aos da zona rural nas facetas autonomia $(\beta=0,12 ; p=0,020)$ e atividades passadas, presentes e futuras $(\beta=0,12$; $\mathrm{p}=0,022$ ), Tabela 3 .

Os idosos da zona rural apresentaram menores escores na faceta funcionamento dos sentidos em relação aos da zona urbana. As limitações oriundas da diminuição do funcionamento sensorial podem interferir nas interações sociais do idoso. Assim, é salutar que os profissionais de saúde identifiquem essas perdas, naturais no processo de envelhecimento, procurando diferenciálas de alterações patológicas, intervindo precocemente ${ }^{27}$. Na zona rural, as perdas dos sentidos são preocupantes, principalmente porque podem trazer maiores agravos devido às características físicas do ambiente, muitas vezes, inadequadas às necessidades dos octogenários.

O escore superior na faceta autonomia entre os octogenários da zona rural pode representar que a capacidade de tomar decisões se encontra preservada, havendo o sentimento de que se sentem úteis, ouvidos e respeitados pela sociedade ${ }^{28}$. Em contrapartida, as condições de saúde inferiores entre os idosos da zona urbana podem ter implicações como a maior dependência de outras pessoas, interferindo também na autonomia e repercutindo nos menores escores nessa faceta.

Os octogenários urbanos apresentaram escore médio inferior aos da zona rural na faceta atividades passadas, presentes e futuras. Nessa faceta é avaliada a satisfação do idoso pelas conquistas e reconhecimento que obteve na vida e as perspectivas futuras ${ }^{12}$. É possível que a situação de saúde dos idosos urbanos, aliada às alterações inerentes ao processo de envelhecimento, não esteja favorecendo anseios futuros. Nesse sentido, é importante que a equipe de saúde junto aos familiares incentive o idoso a buscar novas realizações na vida.

TABELA 3: Comparação dos escores de QV do WHOQOL-BREF e WHOQOL-OLD, segundo local de moradia e análise ajustada por sexo. Uberaba, Minas Gerais, 2008 e 2011.

\begin{tabular}{lcccccccc}
\hline Escores de QV & \multicolumn{2}{c}{ Urbana } & \multicolumn{2}{c}{ Rural } & t & p & $\beta$ & $\mathbf{p}^{(*)}$ \\
& Média & IDP & Média & \pm DP & & & & \\
\hline WHOQOL-BREF & & & & & & & & \\
Físico & 55,47 & 16,77 & 63,17 & 16,79 & $-3,564$ & $<0,001$ & 0,15 & $0,002^{(* *)}$ \\
Psicológico & 65,75 & 11,52 & 68,97 & 14,66 & $-1,77$ & 0,08 & - & - \\
Relações sociais & 68,17 & 10,14 & 71,5 & 12,64 & $-2,432$ & 0,037 & 0,11 & $0,025^{(* *)}$ \\
Meio ambiente & 63,42 & 10,48 & 60,59 & 12,35 & 2,02 & 0,044 & $-0,1$ & $0,036^{(* *)}$ \\
WHOQOL-OLD & & & & & & & & \\
Funcionamento dos sentidos & 75,81 & 21,56 & 69,59 & 22,26 & 2,223 & 0,027 & $-0,11$ & $0,029^{(* *)}$ \\
Autonomia & 58,89 & 12,78 & 63,51 & 17,58 & $-2,135$ & 0,035 & 0,12 & $0,020^{(* *)}$ \\
Atividades passadas, presentes e futuras & 65,88 & 10,44 & 69 & 11,44 & 0,153 & 0,023 & 0,12 & $0,022^{(* *)}$ \\
Participação social & 62,59 & 15,36 & 65,62 & 14,11 & $-1,553$ & 0,121 & - & - \\
Morte e morrer & 78,61 & 22,69 & 74,15 & 24,13 & 1,508 & 0,132 & - & - \\
Intimidade & 68,36 & 15,87 & 72,46 & 17,4 & $-1,969$ & 0,05 & - & - \\
\hline
\end{tabular}

${ }^{(*)} p$ (Ajustado por sexo, empregando-se regressão linear múltipla); ${ }^{(* *)}(p<0,05)$. 


\section{ConClusão}

No presente estudo verificou-se predomínio de mulheres octogenárias residindo na zona urbana e de homens na zona rural. Após o ajuste para a variável de controle, o estado conjugal e o nível de escolaridade permaneceram associados ao local de moradia, sendo que houve maior proporção de idosos na zona urbana que não tinham companheiro em relação aos da zona rural. Em contrapartida, houve proporção superior de idosos sem escolaridade na zona rural.

Em relação às morbidades, os octogenários da zona urbana revelaram piores condições de saúde, referindo em maior proporção má circulação, problemas cardíacos, prisão de ventre e problema de visão que aqueles da zona rural.

Referente à QV, os octogenários da zona urbana apresentaram escores inferiores nos domínio físico e relações sociais e nas facetas autonomia e atividades passadas, presentes e futuras. Por outro lado, os octogenários da zona rural obtiveram escores inferiores aos da zona urbana no domínio meio ambiente e na faceta funcionamento dos sentidos.

Esses resultados apontam para a necessidade de um novo olhar dos serviços de saúde aos octogenários, considerando o ambiente em que vivem. Verificou-se que alguns aspectos foram mais impactados na zona urbana, como a situação de saúde e a QV. Porém, os octogenários que residem na zona rural também enfrentam alguns impasses, relacionados aos fatores socioeconômicos e aos domínios e facetas da QV.

Os profissionais de saúde devem se preparar para $\mathrm{o}$ atendimento a essa parcela dos idosos longevos, com vistas a favorecer não apenas maior longevidade, mas também a QV. Nesse sentido, o acompanhamento das ESF faz-se primordial para a identificação de fatores relacionados ao ambiente em que residem, que possam interferir na situação de saúde e na QV do idoso, de maneira a intervir precocemente.

Ainda que esse estudo apresente limitações, como a não confirmação diagnóstica das morbidades autorreferidas e o delineamento transversal, que não permite estabelecer as relações de causa e efeito entre as variáveis, possibilitou ampliar o conhecimento sobre as características peculiares dessa clientela, considerando a interferência de fatores relacionados ao local de moradia.

\section{REFERÊNCIAS}

1.Instituto Brasileiro de Geografia e Estatística. Projeção da população do Brasil por sexo e idade para o período de 1980-2050. Rio de Janeiro: Ministério do Planejamento, Orçamento e Gestão, 2007. [citado em 28 out 2012] Disponível em: http://www.ibge.gov.br/home/estatistica/ populacao/projecao_da_populacao/default.shtm.
2.Instituto Brasileiro de Geografia e Estatística. Síntese dos indicadores sociais: uma análise das condições de vida da população brasileira. Rio de Janeiro: Ministério do Planejamento, Orçamento e Gestão, 2010. [citado em 28 out 2012] Disponível em: http://www.ibge.gov. br/home/estatistica/populacao/condicaodevida/indicadoresminimos/sinteseindicsociais2010/SIS_2010.pdf.

3.Kirkwood TBL. A systematic look at an old problem: as life expectancy increases, a systems-biology approach is needed to ensure that we have a healthy old age. Nature. 2008; 451:644-7.

4.Ministério da Saúde (Br). DATASUS. Informações de Saúde. 2010. [citado em 02 nov 2012] Disponível em: http://tabnet.datasus.gov.br/cgi/tabcgi.exe?ibge/ cnv/popmg.def.

5.Morais EP, Rodrigues RAP, Gerhardt TE. Os idosos mais velhos no meio rural: realidade de vida e saúde de uma população do interior gaúcho. Texto Contexto Enferm. 2008; 17:374-83.

6.Martins CR, Albuquerque FJB, Gouveia CNNA, Rodrigues CFF, Neves MTS. Avaliação da qualidade de vida subjetiva dos idosos: uma comparação entre os residentes em cidades rurais e urbanas. Estud interdiscip envelhec. 2007; 11:135-54.

7.Rosset I, Roriz-Cruz M, Santos JLF, Haas VJ, FabrícioWehbe SCC, Rodrigues RAP. Diferenciais socioeconômicos e de saúde entre duas comunidades de idosos longevos. Rev Saúde Pública. 2011; 45:391-400.

8.Icaza MC, Albala C. Projeto SABE: Minimental State Examination (MMSE) del estudio de dementia en Chile: análisis estatístico. OPAS; 1999.

9. Bertolucci PHF, Brucki SMD, Campacci SR, Juliano Y. O mini-exame do estado mental em uma população geral: impacto da escolaridade. Arq Neuro-Psiquiatr. 1994; $52(1): 1-7$.

10.Ramos LR. Growing old in São Paulo, Brazil: assessment of health status and family support of the elderly of different socio-economic strata living in the community [these doctor]. London (UK): London School of Hygiene and Tropical Medicine; 1987.

11.Fleck MPA, Louzada S, Xavier M, Chachamovich E, Vieira G, Santos L, et al. Aplicação da versão em português do instrumento abreviado de avaliação da qualidade de vida WHOQOL-bref. Rev Saude Publica. 2000; 34:178-83.

12.Fleck MPA, Chachamovich E, Trentini C. Development and validation of the Portuguese version of the WHOQOLOLD module. Rev Saude Publica. 2006; 40:785-91.

13.Schmidt JA, Dal-Pizzol F, Xavier FM, Heluany V. Aplicação do teste do relógio em octogenários e nonagenários participantes de estudo realizado em Siderópolis/ SC. PSICO. 2009; 40:525-30.

14.Cabral SOL, Oliveira CCC, Vargas MM, Neves ACS. Condições de ambiente e saúde em idosos residentes nas zonas rural e urbana em um município da região nordeste. Geriatria \& Gerontologia. 2010; 4(2):76-84.

15. Aires M, Paskulin LMG, Morais EP. Capacidade funcional de idosos mais velhos: estudo comparativo em três regiões do Rio Grande do Sul. Rev. Latino-Am Enferm. 2010; 18:1-7. 
16.Inouye K, Pedrazzani ES. Nível de instrução, status socioeconômico e avaliação de algumas dimensões da qualidade de vida de octogenários. Rev Latino-Am Enfermagem. 2007; 15:742-7.

17.Santos AS, Karsch UM, Montañés CM. A rede de serviços de atenção à saúde do idoso na cidade de Barcelona (Espanha) e na cidade de São Paulo (Brasil). Ser Soc Soc. 2010; 102:365-86.

18.Ferrari DC, Monteiro ML, Malagutti W, Barnabe AS, Ferraz RRN. Prevalência de lesões cutâneas em pacientes atendidos pelo programa de internação domiciliar (PID) no município de Santos - SP. Conscientiae Saúde. 2010; 9(1):25-32.

19.Santos RFFN, Porfírio GJM, Pitta GBB. A diferença na qualidade de vida de pacientes com doença venosa crônica leve e grave. J Vasc Bras. 2009; 8(2):143-7.

20.Collete VL, Araújo CL, Madruga SW. Prevalência e fatores associados à constipação intestinal: um estudo de base populacional em Pelotas, Rio Grande do Sul, Brasil, 2007. Cad Saúde Pública. 2010; 26:1391-402.

21.Nesello LAN, Tonelli FO, Beltrame TB. Constipação intestinal em idosos frequentadores de um Centro de Convivência no município de Itajaí-SC. CERES. 2011; 6(3):151-62.

22. Yokota RTC, Iser BPM, Andrade RLM, Santos J, Meiners M, Marie MA, et al. Vigilância de fatores de risco e proteção para doenças e agravos não transmissíveis em município de pequeno porte, Brasil, 2010. Epidemiol Serv Saúde. 2010; 21(1):55-68.

23. Soares DA, Toledo JAS, Santos LF, Lima RMB, Galdeano LE. Qualidade de vida de portadores de insuficiência cardíaca. Acta Paul Enferm. 2008; 21:243-8. 24.Ministério da Saúde $(\mathrm{Br})$. Secretaria de Atenção à Saúde. Departamento de Atenção Básica. Cadernos de envelhecimento e saúde da pessoa idosa. Brasília (DF): Ministério da Saúde; 2007.

25.Braga MCP, Casella MA, Campos MLN, Paiva SP. Qualidade de vida medida pelo Whoqol-Bref: estudo com idosos residentes em Juiz de Fora/MG. Rev APS. 2011; 14(1):93-100.

26.Rodrigues LR, Melo e Silva AT, Ferreira PCS, Dias FA, Tavares DMS. Qualidade de vida de idosos com indicativo de depressão: implicações para a enfermagem. Rev enferm UERJ. 2012; 20(esp.2):777-83.

27. Tavares DMS, Gomes NC, Dias FA, Santos NMF. Fatores associados à qualidade de vida de idosos com osteoporose residentes na zona rural. Esc Anna Nery. 2012; 16:371-8.

28.Maués CR, Paschoal SMP, Jaluul O, França CC, Jacob Filho W. Avaliação da qualidade de vida: comparação entre idosos jovens e muito idosos. Rev Bras Clin Med. 2010; 8:405-10. 